\title{
RESISTÊNCIA INDUZIDA EM FRUTOS DE VIDEIRA 'ISABEL' (Vitis labrusca) E SEUS EFEITOS SOBRE A QUALIDADE PÓS-COLHEITA ${ }^{1}$
}

\author{
KEDMA MARIA SILVA PINTO², LUCIANA CORDEIRO DO NASCIMENTO ${ }^{3}$, \\ ANDREZZA KLYVIA OLIVEIRA ${ }^{4}$, RODRIGO PEREIRA LEITE ${ }^{5}$, JULIANA PEREIRA DA SILVA ${ }^{6}$
}

RESUMO-A indução de resistência tem sido apontada como alternativa sustentável para o manejo de doenças, mas pouco se sabe sobre a interferência de tais produtos na qualidade pós-colheita dos frutos. Assim, o presente trabalho tem por objetivo avaliar a produção e a maturação de frutos de videira 'Isabel' (Vitis labrusca), oriundos de plantas tratadas com elicitores de resistência, em Natuba-PB. Os experimentos foram realizados nos períodos de setembro de 2009 a janeiro de 2010 (safra 1) e de fevereiro a junho de 2010 (safra 2). Foram utilizados oito tratamentos (Testemunha; Fungicida (Metiram + Piraclostrobina); Fosfito de potássio; Agro-Mós ${ }^{\circledR}$; Fungicida + Fosfito de potássio; Fungicida + Agro-Mós ${ }^{\circledR}$; Fosfito de potássio + Agro-Mós ${ }^{\circledR}$ e Fungicida + Fosfito de potássio + Agro-Mós $^{\circledR}$ ) e quatro repetições de 5 plantas. As aplicações foram realizadas a cada 10 dias, iniciando-se 20 dias após a poda, totalizando 12 aplicações. As coletas de frutos foram realizadas aos 45; 60;90 e 120 dias após a poda. As variáveis analisadas foram: massa dos cachos, comprimento e diâmetro dos cachos, rendimento de polpa, sólidos solúveis (SS), pH, acidez titulável (AT) e relação SS/AT. O tratamento com fosfito de potássio promoveu o aumento de $24 \%$ no comprimento dos cachos, em relação à testemunha, na primeira safra. O rendimento de polpa e a relação SS/AT não foram influenciados pelos tratamentos, e o teor de sólidos solúveis foi influenciado positivamente pela aplicação de fosfito de potássio, com incrementos superiores a $60 \%$, em ambas as safras avaliadas.

Termos para indexação: Plasmopara viticola, indução de resistência, produção, maturação.

\section{INDUCED RESISTANCE IN FRUITS OF 'ISABEL'VINE (Vitis labrusca) AND ITS EFFECTS ON POST-HARVEST QUALITY}

\begin{abstract}
Induction of resistance has been identified as a sustainable alternative for disease management, but little information is known about the interference of such products in the postharvest fruit. Thus, the present study aims to evaluate the production and ripening of grapes 'Isabel' (Vitis labrusca) from plants treated with resistance elicitors in Natuba-PB, Brazil. The experiments were carried in two seasons, between September 2009 and January 2010 (season 1) and February to June 2010 (season 2). It was used eight treatments (Control; Fungicide (Metiram + Pyraclostrobin); Potassium phosphite; Agro-Mos ${ }^{\circledR}$, Fungicide + Potassium phosphite; Fungicide + Agro-Mos $^{\circledR}$, Potassium phosphite + Agro-Mos $^{\circledR}$ and Fungicide + Potassium phosphite + Agro-Mos $^{\circledR}$ ) with four replications of five plants. The pulverizations were made every 10 days, beginning 20 days after pruning in total of 12 applications. The collected fruits were held at 45, 60, 90 and 120 days after pruning. The variables evaluated were: bunch weight, length and diameter of the clusters, and pulp yield, soluble solids (SS), $\mathrm{pH}$, total acidity (TA) and SS/TA ratio. The treatment with Potassium phosphite promoted a $24 \%$ increase on bunches length, compared to control at season 1 . The pulp yield and harvest index were not affected by treatments and soluble solids content was positively influenced by the application of potassium phosphite, with increases exceeding $60 \%$ in both seasons.
\end{abstract}

Index terms: Plasmopara viticola, resistance induction, production, maturation.

\footnotetext{
1(Trabalho 103-12). Recebido em: 28-02-2012. Aceito para publicação em: 06-02-2013.

${ }^{2}$ Doutoranda em Agronomia - Agricultura Tropical. Programa de Pós-Graduação em Agronomia (PPGA) da Universidade Federal da Paraíba, Centro de Ciências Agrárias (UFPB/CCA). Areia-PB. E-mail: kedma_maria@hotmail.com;

${ }^{3}$ Professor Adjunto. PPGA-UFPB/CCA. Areia-PB. E-mail: luciana.cordeiro@cca.ufpb.br;

${ }^{4}$ Graduanda em Agronomia - UFPB/CCA. Areia-PB. E-mail: andrezza klyvia@hotmail.com;

${ }^{5}$ Mestre em Agronomia - Agricultura Tropical. PPGA-UFPB/CCA. Areia-PB. E-mail: leiterp@hotmail.com;

${ }^{6}$ Mestre em Agronomia - Agricultura Tropical. PPGA-UFPB/CCA. Professora Faculdade da Amazônia - FAMA. Vilhena-RO. E-mail: julaip@hotmail.com
} 


\section{INTRODUÇÃO}

O cultivo de videira assume um importante papel no contexto social e econômico a nível mundial, sendo uma das principais frutíferas cultivadas. Itália e China são os maiores produtores,e o Brasil ocupa a $15^{\mathrm{a}}$ posição no ranking mundial, com 1.365.491 toneladas em 2009, movimentando um mercado exportador de US\$ 171 milhões, sendo as regiões Sul e Nordeste as maiores produtoras (FAO, 2011; IBGE, 2011).

A videira 'Isabel' é uma das principais cultivares de Vitis labrusca L., espécie originária do sul dos Estados Unidos e de onde foi difundida para outras regiões, destinada principalmente à indústria e apresenta normalmente cachos com tamanho e peso inferiores quando comparada a outras cultivares, tais como Itália e Benitaka; no entanto, mostra-se com maiores valores de sólidos solúveis (MASCARENHAS et al., 2010).

O manejo de doenças em parreirais paraibanos ainda se constitui em um dos principais problemas para a região, visto que é realizado com o uso intensivo de fungicidas, e, por este motivo, a indução de resistência surge como alternativa promissora para o controle de doenças (GOMES, 2009).

O fenômeno da indução de resistência de plantas contra patógenos está relacionado com a ativação de variados mecanismos de defesa através de elicitores ou indutores (DAVID et al., 2010). Sendo assim, tal processo envolve uma série de reações bioquímicas e fisiológicas, podendo gerar modificações em seu metabolismo e na formação dos frutos. Estes elicitores são organismos bióticos e abióticos, de acordo com seu modo de ação, e desencadeiam processos de defesa com ação sistêmica ou localizada (DIAS; RANGEL, 2007).

As plantas, ao se depararem com uma ampla gama de estímulos externos, desencadeiam a produção de diversos metabólitos secundários, que se acumulam em tipos de células especializadas ou fora da planta, em paredes celulares, sobre a folha ou na rizosfera das plantas. Assim, sofrem modificações enzimáticas e alteram as propriedades fisiológicas e bioquímicas dessas moléculas, alterando a sua função e localização dentro da planta (HALL et al., 2011).

Muito embora alguns elicitores de resistência já estejam sendo testados quanto a sua eficiência nesta região, baseando-se na premissa de que tal fenômeno pode ser desencadeado através de produtos menos agressivos ao homem e ao meio ambiente, e de custo reduzido (ROSA et al., 2006; GOMES, 2009), ainda pouco se sabe sobre a interferência de tais produtos na qualidade dos frutos.
O presente trabalho teve por objetivo avaliar o aspecto de produção e maturação de frutos de uva 'Isabel' oriundos de plantas tratadas com elicitores de resistência, no município de Natuba-PB.

\section{MATERIAL E MÉTODOS}

Os experimentos foram realizados em área comercial de produção de videiras 'Isabel', plantadas com espaçamento de $1,5 \times 1,5 \mathrm{~m}$, com área experimental de $2.500 \mathrm{~m}^{2}$, localizadas no município de Natuba - PB, no Sítio Fervedouro ( $35^{\circ} 32^{\prime} \mathrm{W}$. e $07^{\circ} 35^{\prime} \mathrm{S}$.), em duas safras, no período de outubro de 2009 a fevereiro de 2010 (safra 1), e de fevereiro a junho de 2010 (safra 2). As análises de qualidade foram realizadas no Laboratório de Fitopatologia da Universidade Federal da Paraíba - Centro de Ciências Agrárias (UFPB-CCA).

As aplicações com os elicitores foram realizadas a cada dez dias, iniciando-se estas 20 dias após a poda das videiras, utilizando-se de pulverizador costal motorizado atomizador, com capacidade de $13 \mathrm{~L}$, perfazendo um total de doze aplicações, num período de quatro meses. O delineamento estatístico em blocos ao acaso constituiu-se de oito tratamentos: Testemunha; Fungicida (Metiram + Piraclostrobina); Fosfito de potássio; Agro-Mós ${ }^{\circledR}$; Fungicida + Fosfito de potássio; Fungicida + Agro-Mós $^{\circledR}$; Fosfito de potássio + Agro-Mós ${ }^{\circledR}$ e Fungicida + Fosfito de potássio + Agro-Mós ${ }^{\circledR}$ e quatro repetições, sendo a unidade amostral composta por três cachos retirados aleatoriamente das cinco plantas que compunham cada tratamento .

As coletas de uvas foram realizadas aos 45; 60; 90 e 120 dias após a poda (DAP) para análises físico-químicas. Os atributos físicos de qualidade avaliados foram a massa dos cachos $(\mathrm{g})$ mediante balança analítica, o comprimento e diâmetro dos cachos (mm), mensurados com paquímetro digital, e rendimento de polpa $(\mathrm{g})$, avaliado a partir da extração da casca, semente e engaço.

As análises físico-químicas foram determinadas a partir do mosto obtido através do desengace e esmagamento das bagas, para sólidos solúveis SS (\%), pH, acidez titulável e relação SS/AT (AOAC, 1984). Os dados obtidos foram submetidos à análise de variância, e as médias, comparadas pelo teste de Tukey, a 5\% de probabilidade, pelo programa estatístico ASSISTAT (ASSISTAT, 2010).

Também foram realizados acompanhamento diário dos dados climáticos e monitoramento de doenças, como a antracnose (Elsinoe ampelina) e o míldio (Plasmopara viticola), sendo a unidade amostral constituída de cinco plantas, sendo retiradas 
para análise nove folhas de cada planta (três folhas basais, três medianas e três apicais).

\section{RESULTADOS E DISCUSSÃO}

Para os atributos de qualidade física de frutos, o Fosfito de potássio proporcionou frutos com maior massa, diferindo apenas da testemunha e do Agro-Mós ${ }^{\circledR}$, aos 120 DAP na primeira safra (Tabela 1).

Em relação à massa dos cachos, na safra 1 , as uvas de plantas tratadas com fosfito de potássio obtiveram maiores massas, com média final de $144,62 \mathrm{~g}$, diferindo apenas da testemunha $(91,87 \mathrm{~g})$ e das plantas tratadas com Agro-Mós ${ }^{\circledR}(99,50 \mathrm{~g})$, aos 120 dias após a poda (DAP). O Agro-Mós ${ }^{\circledR}$, a partir dos 60 dias, apresentou os menores valores para esta variável, diferindo apenas do fosfito de potássio e fungicida + fosfito de potássio neste período ou apenas do fosfito de potássio aos 90 e 120 DAP.

$\mathrm{Na}$ safra 2, os tratamentos não influenciaram a massa dos cachos até os 90 DAP. Os frutos com maior massa final foram os colhidos de plantas tratadas com fungicida, único tratamento que diferiu da testemunha aos 120 DAP e obteve uma média de $148,1 \mathrm{~g}$. Tal fato pode ser decorrente da ação fungicida contra as doenças, resultando em cacho com menor índice de aborto de flores, maior número de bagas/ cacho e, consequentemente, maior massa do cacho.

Gomes (2009) verificou diferença significativa na massa dos cachos de uva "Isabel' tratadas com elicitores de resistência, em Natuba-PB, onde o uso de fosfito de potássio e Rocksil ${ }^{\circledR}$ não diferiu de plantas tratadas com fungicidas.

O fosfito de potássio promoveu ,além de maior acúmulo de massa nas uvas, desde a formação dos frutos até a colheita, acréscimo superior a $24 \%$ no tamanho final dos cachos em relação à testemunha (Tabela 2) na primeira safra, não diferindo do tratamento com fungicida, fungicida + Agro-Mós $^{\circledR}$ e Fosfito + Agro-Mós ${ }^{\circledR}$.

Segundo informações técnicas do fosfito de potássio, a aplicação de fosfito é sugerida para todo tipo de cultivo por favorecer a prevenção e a cura dos sintomas produzidos por fungos; no entanto, seu uso associa-se principalmente à melhoria do estado nutricional das plantas, sobretudo nos estádios de maior aumento da atividade metabólica, favorecendo o amadurecimento e a qualidade dos frutos, além de qualidade superior na pós-colheita (OLIVEIRA, 2007).

Na safra 2, apenas aos 120 DAP, os tratamentos influenciaram no comprimento dos cachos e apenas as plantas tratadas com fungicida diferiram da testemunha.
A utilização de elicitores de resistência dentre outras práticas alternativas são opções ecológicas e promissoras para substituir a proteção tradicional promovida pela aplicação de fungicidas, com a vantagem de poder ser utilizada junto com outras práticas de manejo integrado de doenças, contribuindo para $o$ atendimento à crescente demanda mundial por produtos orgânicos (DIAS-ARIEITA et al., 2010).

Durante os experimentos, foi observada elevada incidência de doenças, principalmente na segunda safra (Tabela 3), o que se atribuiu aos maiores índices de precipitação e umidade (Figura 1), com temperaturas mais baixas.

O uso de Agro-Mós ${ }^{\circledR}$ como elicitor de resistência em videiras 'Isabel' promoveu um desempenho inferior na produção de cachos quando comparados com aqueles oriundos de plantas tratadas com fungicida e/ou fosfito de potássio, principalmente na segunda safra, o que deve, possivelmente, à elevada incidência de doenças, como a antracnose da videira, com 53,3\%, não diferindo da testemunha.

Os maiores diâmetros finais dos cachos foram obtidos pela aplicação de fungicida $(62,2 \mathrm{~mm})$, que diferiu apenas das plantas tratadas com Agro-Mós ${ }^{\circledR}$ $(53,9 \mathrm{~mm})$ e da combinação deste com o fosfito de potássio $(51,2 \mathrm{~mm})$ (Tabela 4) na safra 1. Na safra 2 , não houve diferença significativa entre os tratamentos para esta variável, aos 60; 90 e 120 DAP.

O número de bagas e o rendimento de polpa de uvas 'Isabel' não foram influenciados pela aplicação de elicitores de resistência nas duas safras em que foram realizados os experimentos.

O acúmulo de açúcares nas uvas 'Isabel' não diferiu estatisticamente entre os tratamentos, durante a formação dos frutos, mas apenas no ponto de colheita (120 DAP), em ambas as safras. No entanto, na segunda safra, todos os tratamentos diferiram da testemunha, mas não diferiram entre si e obtiveram maiores médias em relação à safra 1 , com até $18,3^{\circ}$ brix (Tabela 5).

Sato et al. (2009) obtiveram valores máximos de sólidos solúveis de 16,8 e 16,3\% quando avaliados diferentes porta-enxertos para a variedade 'Isabel', na região norte do Paraná. No entanto, os valores médios sugeridos para o mosto desta cultivar giram em torno de 18,6\% de sólidos solúveis (EMBRAPA, 2006).

A acidez titulável (AT) decresceu à medida que aumentou o processo de maturação (Tabela 6), ao contrário do que se observou com os sólidos solúveis. Estes dois atributos de qualidade são importantes para a determinação do índice de colheita, a partir da relação SS/AT. Na safra 1, apenas os tratamentos fungicida + fosfito de potássio + Agro-Mós ${ }^{\circledR}(0,95 \%$ de ácido tartárico) e fungicida + Agro-Mós ${ }^{\circledR}(0,6 \%$ 
de ácido tartárico) diferiram entre si, enquanto na segunda safra não houve influência dos elicitores para esta variável,aos 45; 60; 90 e 120 DAP (Tabela 7).

Os teores de ácido tartárico no mosto, verificados neste ensaio $\left(5,7\right.$ a $\left.9,5 \mathrm{~g} \mathrm{~L}^{-1}\right)$, estão dentro da faixa estabelecida, que varia de 3 a $9 \mathrm{~g} \mathrm{~L}^{-1}$ (EMBRAPA, 2006). Rizzon e Sganzerla (2007) verificaram valores médios de 4,35 $\mathrm{g} \mathrm{L}^{-1}$ de ácido tartárico em uvas Isabel, apresentando teores médios mais elevados nos mostos das cultivares de Vitis vinifera do que nas cultivares da espécie Vitis labrusca.

A evolução da acidez titulável está relacionada ao fato de os principais ácidos das videiras (tartárico e málico) serem sintetizados pelas folhas e pelas bagas ainda verdes;por isso, no início da maturação, as bagas apresentam elevado teor de AT. Com a evolução da maturação, a demanda por energia aumenta e, para suprir essa necessidade, os ácidos são utilizados como fonte de energia na respiração celular ou no acúmulo de carboidratos (BLOUIN; GUIMBERTEAU, 2004; TAIZ; ZEIGER, 2006).

Quanto à variável $\mathrm{pH}$, apenas aos $90 \mathrm{DAP}$, na primeira safra, houve diferença significativa entre o fungicida e o fosfito de potássio, mas estes não diferiram da testemunha nem dos demais tratamentos (Tabela 8). O maior valor registrado foi de 3,15 , aos 120 DAP, enquadrando-se nos valores médios indicados para esta cultivar $(3,27)$ (EMBRAPA, 2006).

$\mathrm{O} \mathrm{pH}$ influencia diretamente na estabilidade das antocianinas, portanto o conhecimento desta característica para cada indutor de resistência é extremamente importante quando se deseja obter sucos com altos teores de matéria corante (MALACRIDA; MOTTA, 2006).

TABELA 1 - Massa dos cachos de uva 'Isabel' (g) de plantas tratadas com elicitores de resistência, no período de setembro de 2009 a fevereiro de 2010 (safra 1) e fevereiro 2010 a junho de 2010 (safra 2). Natuba-PB.

\begin{tabular}{lcccccccc}
\hline & \multicolumn{4}{c}{ Safra 1 } & \multicolumn{4}{c}{ Safra 2 } \\
\hline & $\mathbf{4 5}^{\mathbf{1}}$ & $\mathbf{6 0}$ & $\mathbf{9 0}$ & $\mathbf{1 2 0}$ & $\mathbf{4 5}$ & $\mathbf{6 0}$ & $\mathbf{9 0}$ & $\mathbf{1 2 0}$ \\
\hline Testemunha & $44,9 \mathrm{~b}^{2}$ & $79,1 \mathrm{bc}$ & $85,7 \mathrm{ab}$ & $91,9 \mathrm{~b}$ & $84,9 \mathrm{a}$ & $91,8 \mathrm{a}$ & $97,0 \mathrm{bc}$ & $107,6 \mathrm{~b}$ \\
Fungicida & $62,1 \mathrm{ab}$ & $94,5 \mathrm{abc}$ & $106,9 \mathrm{ab}$ & $123,6 \mathrm{ab}$ & $82,8 \mathrm{a}$ & $87,5 \mathrm{a}$ & $93,0 \mathrm{c}$ & $148,1 \mathrm{a}$ \\
Fosfito & $82,0 \mathrm{a}$ & $107,6 \mathrm{a}$ & $120,3 \mathrm{a}$ & $144,6 \mathrm{a}$ & $80,3 \mathrm{a}$ & $100,6 \mathrm{a}$ & $101,0 \mathrm{abc}$ & $103,8 \mathrm{~b}$ \\
Agro-Mós $^{\circledR}$ & $60,4 \mathrm{ab}$ & $74,7 \mathrm{c}$ & $84,5 \mathrm{~b}$ & $99,5 \mathrm{~b}$ & $83,8 \mathrm{a}$ & $87,1 \mathrm{a}$ & $91,0 \mathrm{c}$ & $100,0 \mathrm{~b}$ \\
Fung+Fosf. & $74,1 \mathrm{a}$ & $101,0 \mathrm{ab}$ & $106,5 \mathrm{ab}$ & $129,8 \mathrm{ab}$ & $85,1 \mathrm{a}$ & $88,9 \mathrm{a}$ & $91,0 \mathrm{c}$ & $104,2 \mathrm{~b}$ \\
Fung.+Agro-Mós $^{\circledR}$ & $63,5 \mathrm{ab}$ & $77,0 \mathrm{c}$ & $81,0 \mathrm{~b}$ & $106,8 \mathrm{ab}$ & $73,3 \mathrm{a}$ & $108,2 \mathrm{a}$ & $111,0 \mathrm{ab}$ & $118,0 \mathrm{ab}$ \\
Fosf.+Agro-Mós $^{\circledR}$ & $71,8 \mathrm{a}$ & $89,1 \mathrm{abc}$ & $96,1 \mathrm{ab}$ & $105,2 \mathrm{ab}$ & $71,7 \mathrm{a}$ & $94,9 \mathrm{a}$ & $108,0 \mathrm{abc}$ & $114,0 \mathrm{ab}$ \\
Fung.+Fosf.+ Agro-Mós $^{\circledR}$ & $77,9 \mathrm{a}$ & $96,9 \mathrm{abc}$ & $90,3 \mathrm{ab}$ & $107,0 \mathrm{ab}$ & $89,0 \mathrm{a}$ & $107,9 \mathrm{a}$ & $117,0 \mathrm{a}$ & $133,0 \mathrm{ab}$ \\
\hline CV (\%) & 16,4 & 10,4 & 15,5 & 15,02 & 17,6 & 9,6 & 18,4 & 14,1 \\
\hline
\end{tabular}

${ }^{1}$ Dias após a poda . ${ }^{2}$ As médias seguidas da mesma letra não diferem estatisticamente entre si, pelo teste de Tukey, a 5\% de probabilidade. Médias de três cachos.

TABELA 2- Comprimento dos cachos de uva 'Isabel' ( $\mathrm{mm}$ ) de plantas tratadas com elicitores de resistência, no período de setembro de 2009 a fevereiro de 2010 (safra 1) e fevereiro 2010 a junho de 2010 (safra 2). Natuba-PB.

\begin{tabular}{|c|c|c|c|c|c|c|c|c|}
\hline & \multicolumn{4}{|c|}{ Safra 1} & \multicolumn{4}{|c|}{ Safra 2} \\
\hline & $45^{1}$ & 60 & 90 & 120 & 45 & 60 & 90 & 120 \\
\hline Testemunha & $81,3 b^{2}$ & $85,7 \mathrm{~b}$ & $90,9 \mathrm{~b}$ & $92,5 \mathrm{bc}$ & $90,3 \mathrm{a}$ & $91,1 \mathrm{a}$ & $93,0 \mathrm{a}$ & $94,9 \mathrm{bcd}$ \\
\hline Fungicida & $80,7 \mathrm{~b}$ & $96,0 \mathrm{ab}$ & $100,5 \mathrm{ab}$ & $108,6 \mathrm{ab}$ & $76,3 \mathrm{a}$ & $86,8 \mathrm{a}$ & $102,0 \mathrm{a}$ & $113,0 \mathrm{a}$ \\
\hline Fosfito & $95,5 \mathrm{a}$ & $106,1 \mathrm{a}$ & $108,5 \mathrm{a}$ & 114,9 a & $88,5 \mathrm{a}$ & $90,2 \mathrm{a}$ & $90,5 \mathrm{a}$ & $90,9 \mathrm{~cd}$ \\
\hline Agro-Mós ${ }^{\circledR}$ & $85,0 \mathrm{ab}$ & $87,5 \mathrm{~b}$ & $88,8 \mathrm{~b}$ & $94,9 \mathrm{bc}$ & $84,6 \mathrm{a}$ & $85,4 \mathrm{a}$ & $86,0 \mathrm{a}$ & $86,8 \mathrm{~d}$ \\
\hline Fung+Fosf. & $90,9 a b$ & $93,1 \mathrm{ab}$ & $95,0 \mathrm{ab}$ & $106,1 \mathrm{bc}$ & $83,5 \mathrm{a}$ & $87,1 \mathrm{a}$ & $93,0 \mathrm{a}$ & $101,0 \mathrm{abc}$ \\
\hline Fung.+Agro-Mós ${ }^{\circledR}$ & $92,9 \mathrm{ab}$ & $97,5 \mathrm{ab}$ & $100,4 \mathrm{ab}$ & $103,7 \mathrm{abc}$ & $76,7 \mathrm{a}$ & $94,2 \mathrm{a}$ & $97,0 \mathrm{a}$ & $100,8 \mathrm{abc}$ \\
\hline Fosf.+Agro-Mós ${ }^{\circledR}$ & $90,6 \mathrm{ab}$ & $92,1 \mathrm{~b}$ & $94,3 \mathrm{~b}$ & $98,3 \mathrm{abc}$ & $81,3 \mathrm{a}$ & $91,3 \mathrm{a}$ & $95,0 \mathrm{a}$ & $100,0 \mathrm{bc}$ \\
\hline Fung.+Fosf.+ Agro-Mós ${ }^{\circledR}$ & $96,3 \mathrm{a}$ & $98,4 \mathrm{ab}$ & $91,3 \mathrm{~b}$ & $90,3 \mathrm{c}$ & $96,8 \mathrm{a}$ & $98,9 \mathrm{a}$ & $101,0 \mathrm{a}$ & $103,8 \mathrm{ab}$ \\
\hline CV (\%) & 5,96 & 5,88 & 6,09 & 7,55 & 12,06 & 7,42 & 12,47 & 5,47 \\
\hline
\end{tabular}

1- Dias após a poda. ${ }^{2}$-As médias seguidas da mesma letra não diferem estatisticamente entre si, pelo teste de Tukey, a 5\% de probabilidade. Médias de 3 frutos. 
TABELA 3- Incidência de Antracnose (Elsinoe ampelina) e Míldio (Plasmopara viticola) (\%) em videiras 'Isabel' tratadas com elicitores de resistência, no período de fevereiro a junho de 2010. Natuba-PB.

\begin{tabular}{|c|c|c|c|c|}
\hline Tratamentos & $\mathbf{A 1} \mathbf{1}^{1}$ & M1 & $\mathbf{A 2}$ & M2 \\
\hline Testemunha & $41,11 \mathrm{a}^{2}$ & $58,90 \mathrm{a}$ & $41,11 \mathrm{a}$ & $\overline{70,37 \mathrm{a}}$ \\
\hline Fungicida (2kg/ha) & $3,89 \mathrm{~b}$ & $11,71 \mathrm{c}$ & $3,89 \mathrm{~b}$ & $28,70 \mathrm{~b}$ \\
\hline Fosfito de Potássio (130g/100L) & $4,47 \mathrm{~b}$ & $13,32 \mathrm{bc}$ & $4,47 \mathrm{~b}$ & $22,22 \mathrm{bc}$ \\
\hline Agro-Mós $\left.{ }^{\circledR}{ }^{(3 m L} / \mathbf{L}\right)$ & $53,33 \mathrm{a}$ & $10,61 \mathrm{c}$ & $53,33 \mathrm{a}$ & $16,67 \mathrm{c}$ \\
\hline Fungicida $(1 \mathrm{~kg} / \mathrm{ha})+$ Fosfito de Potássio $(65 \mathrm{~g} / 100 \mathrm{~L})$ & $4,44 \mathrm{~b}$ & $23,90 \mathrm{~b}$ & $4,44 \mathrm{~b}$ & $25,00 \mathrm{bc}$ \\
\hline Fungicida $(1 \mathrm{~kg} / \mathrm{ha})+\operatorname{AgroMos}^{\circledR}(1,5 \mathrm{~mL} / \mathrm{L})$ & $7,22 \mathrm{~b}$ & $13,94 \mathrm{bc}$ & $7,22 \mathrm{~b}$ & $25,93 \mathrm{bc}$ \\
\hline Fosfito de Potássio (65g/100L) + $\operatorname{AgroMos}^{\circledR}(1,5 \mathrm{~mL} / \mathrm{L})$ & $16,11 \mathrm{~b}$ & $8,92 \mathrm{c}$ & $16,11 \mathrm{~b}$ & $19,44 \mathrm{bc}$ \\
\hline $\begin{array}{l}\text { Fungicida }(0,67 \mathrm{~kg} / \mathrm{ha})+\text { Fosfito de Potássio }(43 \mathrm{~g} / 100 \mathrm{~L})+\text { Agro- } \\
-\mathrm{Mós}^{\circledR}{ }^{\circledR}(1 \mathrm{~mL} / \mathrm{L})\end{array}$ & $5,55 \mathrm{~b}$ & $13,31 \mathrm{bc}$ & $5,55 \mathrm{~b}$ & $25,00 \mathrm{bc}$ \\
\hline CV $(\%)$ & 44,32 & 22,32 & 24,32 & 16,91 \\
\hline
\end{tabular}

${ }^{1} \mathrm{~A}=$ Incidência de Antracnose (\%) nas safras 1 e 2; $\mathrm{M}=$ Incidência de Míldio (\%) nas safras 1 e 2;

${ }^{2}$-As médias seguidas da mesma letra não diferem estatisticamente entre si, pelo teste de Tukey, a 5\% de probabilidade. Médias de 20 plantas.

TABELA 4 - Diâmetro dos cachos ( $\mathrm{mm}$ ) de frutos de uva 'Isabel' colhidos de plantas tratadas com elicitores de resistência, no período de setembro de 2009 a janeiro de 2010 (safra 1) e fevereiro a junho de 2010 (safra 2). Natuba-PB.

\begin{tabular}{lcccccccc}
\hline & \multicolumn{4}{c}{ Safra $\mathbf{1}$} & \multicolumn{4}{c}{ Safra 2 } \\
\hline & $\mathbf{4 5}$ & $\mathbf{6 0}$ & $\mathbf{9 0}$ & $\mathbf{1 2 0}$ & $\mathbf{4 5}$ & $\mathbf{6 0}$ & $\mathbf{9 0}$ & $\mathbf{1 2 0}$ \\
Testemunha & $44,1 \mathrm{~b}$ & $46,3 \mathrm{c}$ & $47,3 \mathrm{c}$ & $58,4 \mathrm{abc}$ & $49,3 \mathrm{ab}$ & $59,7 \mathrm{a}$ & $59,7 \mathrm{a}$ & $59,9 \mathrm{a}$ \\
Fungicida & $49,9 \mathrm{ab}$ & $53,6 \mathrm{ab}$ & $57,1 \mathrm{a}$ & $62,2 \mathrm{a}$ & $49,2 \mathrm{ab}$ & $59,2 \mathrm{a}$ & $59,4 \mathrm{a}$ & $59,9 \mathrm{a}$ \\
Fosfito & $52,8 \mathrm{a}$ & $53,8 \mathrm{ab}$ & $55,4 \mathrm{ab}$ & $60,5 \mathrm{ab}$ & $47,1 \mathrm{ab}$ & $56,6 \mathrm{a}$ & $56,3 \mathrm{a}$ & $55,2 \mathrm{a}$ \\
Agro-Mós ${ }^{\circledR}$ & $49,3 \mathrm{ab}$ & $49,9 \mathrm{bc}$ & $52,3 \mathrm{abc}$ & $53,9 \mathrm{bc}$ & $54,0 \mathrm{ab}$ & $54,5 \mathrm{a}$ & $55,0 \mathrm{a}$ & $56,7 \mathrm{a}$ \\
Fung+Fosf. & $52,6 \mathrm{a}$ & $56,0 \mathrm{a}$ & $56,5 \mathrm{ab}$ & $58,2 \mathrm{abc}$ & $51,7 \mathrm{ab}$ & $59,6 \mathrm{a}$ & $56,9 \mathrm{a}$ & $55,5 \mathrm{a}$ \\
Fung.+Agro-Mós ${ }^{\circledR}$ & $46,8 \mathrm{ab}$ & $50,5 \mathrm{abc}$ & $52,7 \mathrm{abc}$ & $55,4 \mathrm{abc}$ & $45,8 \mathrm{~b}$ & $60,6 \mathrm{a}$ & $58,9 \mathrm{a}$ & $56,6 \mathrm{a}$ \\
Fosf.+Agro-Mós & $48,3 \mathrm{ab}$ & $49,0 \mathrm{bc}$ & $50,0 \mathrm{bc}$ & $51,2 \mathrm{c}$ & $55,1 \mathrm{a}$ & $59,0 \mathrm{a}$ & $57,5 \mathrm{a}$ & $56,5 \mathrm{a}$ \\
Fung.+Fosf.+HgroMós ${ }^{\circledR}$ & $50,4 \mathrm{ab}$ & $51,7 \mathrm{abc}$ & $53,7 \mathrm{abc}$ & $58,7 \mathrm{abc}$ & $52,8 \mathrm{ab}$ & $60,0 \mathrm{a}$ & $60,1 \mathrm{a}$ & $60,0 \mathrm{a}$ \\
\hline $\mathbf{C V ( \% )}$ & $\mathbf{5 , 9 5}$ & $\mathbf{4 , 8 7}$ & $\mathbf{5 , 3 1}$ & $\mathbf{7 , 8 2}$ & $\mathbf{7 , 3 9}$ & $\mathbf{4 , 3 3}$ & $\mathbf{6 , 7 8}$ & $\mathbf{5 , 3 9}$ \\
\hline
\end{tabular}

*Médias seguidas da mesma letra não diferem estatisticamente entre si, pelo teste de Tukey, a 5\% de probabilidade. Médias de 3 cachos. Fung=fungicida; Fosf. $=$ fosfito de potássio.

TABELA 5- Teor de sólidos solúveis (\%) de frutos de videira 'Isabel' tratados com elicitores de resistência, no período de setembro de 2009 a janeiro de 2010 (safra 1) e fevereiro a junho de 2010 (safra 2). Natuba-PB.

\begin{tabular}{lccccccccc}
\hline & \multicolumn{4}{c}{ Safra 1 } & \multicolumn{4}{c}{ Safra 2 } \\
\hline & $\mathbf{4 5}^{\mathbf{1}}$ & $\mathbf{6 0}$ & $\mathbf{9 0}$ & $\mathbf{1 2 0}$ & $\mathbf{4 5}$ & $\mathbf{6 0}$ & $\mathbf{9 0}$ & $\mathbf{1 2 0}$ \\
\hline Testemunha & $4,0 \mathrm{a}^{2}$ & $5,0 \mathrm{a}$ & $6,9 \mathrm{a}$ & $10,4 \mathrm{c}$ & $4,9 \mathrm{a}$ & $5,8 \mathrm{a}$ & $7,4 \mathrm{a}$ & $11,23 \mathrm{~b}$ \\
Fungicida & $3,9 \mathrm{a}$ & $4,9 \mathrm{a}$ & $6,9 \mathrm{a}$ & $10,8 \mathrm{c}$ & $5,1 \mathrm{a}$ & $5,9 \mathrm{a}$ & $8,9 \mathrm{a}$ & $16,88 \mathrm{a}$ \\
Fosfito & $3,9 \mathrm{a}$ & $4,8 \mathrm{a}$ & $7,0 \mathrm{a}$ & $11,8 \mathrm{bc}$ & $4,4 \mathrm{a}$ & $5,6 \mathrm{a}$ & $8,3 \mathrm{a}$ & $15,8 \mathrm{a}$ \\
Agro-Mós ${ }^{\circledR}$ & $4,1 \mathrm{a}$ & $5,0 \mathrm{a}$ & $7,0 \mathrm{a}$ & $16,5 \mathrm{a}$ & $5,0 \mathrm{a}$ & $5,8 \mathrm{a}$ & $8,9 \mathrm{a}$ & $17,18 \mathrm{a}$ \\
Fung+Fosf. & $4,4 \mathrm{a}$ & $4,8 \mathrm{a}$ & $6,7 \mathrm{a}$ & $11,0 \mathrm{c}$ & $5,2 \mathrm{a}$ & $5,7 \mathrm{a}$ & $9,5 \mathrm{a}$ & $17,3 \mathrm{a}$ \\
Fung.+Agro-Mós ${ }^{\circledR}$ & $3,9 \mathrm{a}$ & $5,1 \mathrm{a}$ & $7,0 \mathrm{a}$ & $11,1 \mathrm{c}$ & $4,9 \mathrm{a}$ & $6,0 \mathrm{a}$ & $9,0 \mathrm{a}$ & $18,27 \mathrm{a}$ \\
Fosf.+Agro-Mós ${ }^{\circledR}$ & $4,1 \mathrm{a}$ & $5,2 \mathrm{a}$ & $7,1 \mathrm{a}$ & $15,1 \mathrm{ab}$ & $5,0 \mathrm{a}$ & $6,1 \mathrm{a}$ & $8,7 \mathrm{a}$ & $18,01 \mathrm{a}$ \\
Fung.+Fosf.+Agro-Mós ${ }^{\circledR}$ & $4,0 \mathrm{a}$ & $4,8 \mathrm{a}$ & $6,9 \mathrm{a}$ & $14,9 \mathrm{ab}$ & $4,9 \mathrm{a}$ & $5,8 \mathrm{a}$ & $9,9 \mathrm{a}$ & $17,27 \mathrm{a}$ \\
\hline $\mathbf{C V ~ ( \% )}$ & $\mathbf{6 , 1 1}$ & $\mathbf{7 , 0 6}$ & $\mathbf{6 , 8 9}$ & $\mathbf{1 0 , 9 9}$ & $\mathbf{5 , 5 6}$ & $\mathbf{4 , 0 2}$ & $\mathbf{1 0 , 9 6}$ & $\mathbf{1 2 , 2}$ \\
\hline
\end{tabular}

Dias após a poda

As médias seguidas da mesma letra não diferem estatisticamente entre si, pelo teste de Tukey, a 5\% de probabilidade. Médias de 3 frutos. 
TABELA 6- Acidez titulável (\% de ácido tartárico) de frutos de videira 'Isabel' tratados com indutores de resistência, no período de setembro de 2009 a janeiro de 2010 (safra 1) e de fevereiro a junho de 2010 (safra 2). Natuba-PB.

\begin{tabular}{lcccccccc}
\hline & \multicolumn{4}{c}{ Safra 1 } & \multicolumn{5}{c}{ Safra 2 } \\
\hline & $\mathbf{4 5}^{\mathbf{1}}$ & $\mathbf{6 0}$ & $\mathbf{9 0}$ & $\mathbf{1 2 0}$ & $\mathbf{4 5}$ & $\mathbf{6 0}$ & $\mathbf{9 0}$ & $\mathbf{1 2 0}$ \\
Testemunha & $2,83 \mathrm{ab}$ & $2,36 \mathrm{ab}$ & $2,22 \mathrm{a}$ & $0,87 \mathrm{ab}$ & $2,40 \mathrm{abc}$ & $1,99 \mathrm{a}$ & $1,55 \mathrm{a}$ & $0,74 \mathrm{a}$ \\
Fungicida & $2,40 \mathrm{~b}$ & $2,17 \mathrm{abc}$ & $2,29 \mathrm{a}$ & $0,70 \mathrm{ab}$ & $2,24 \mathrm{c}$ & $1,82 \mathrm{a}$ & $1,59 \mathrm{a}$ & $0,54 \mathrm{~b}$ \\
Fosfito & $2,45 \mathrm{~b}$ & $2,27 \mathrm{abc}$ & $2,25 \mathrm{a}$ & $0,67 \mathrm{ab}$ & $2,37 \mathrm{bc}$ & $1,90 \mathrm{a}$ & $1,71 \mathrm{a}$ & $0,56 \mathrm{~b}$ \\
Agro-Mós ${ }^{\circledR}$ & $2,50 \mathrm{~b}$ & $2,10 \mathrm{bc}$ & $2,25 \mathrm{a}$ & $0,88 \mathrm{ab}$ & $2,40 \mathrm{abc}$ & $1,74 \mathrm{a}$ & $1,58 \mathrm{a}$ & $0,74 \mathrm{a}$ \\
Fung+Fosf. & $3,01 \mathrm{a}$ & $2,43 \mathrm{a}$ & $2,60 \mathrm{a}$ & $0,81 \mathrm{ab}$ & $2,74 \mathrm{a}$ & $2,12 \mathrm{a}$ & $1,90 \mathrm{a}$ & $0,71 \mathrm{a}$ \\
Fung.+Agro-Mós ${ }^{\circledR}$ & $2,73 \mathrm{ab}$ & $1,98 \mathrm{c}$ & $2,16 \mathrm{a}$ & $0,60 \mathrm{~b}$ & $2,47 \mathrm{abc}$ & $1,77 \mathrm{a}$ & $1,51 \mathrm{a}$ & $0,51 \mathrm{~b}$ \\
Fosf.+Agro-Mós & $2,60 \mathrm{ab}$ & $2,28 \mathrm{abc}$ & $2,58 \mathrm{a}$ & $0,81 \mathrm{ab}$ & $2,59 \mathrm{ab}$ & $2,02 \mathrm{a}$ & $1,95 \mathrm{a}$ & $0,70 \mathrm{a}$ \\
Fung.+Fosf.+ Agro-Mós ${ }^{\circledR}$ & $2,73 \mathrm{ab}$ & $2,09 \mathrm{bc}$ & $2,62 \mathrm{a}$ & $0,95 \mathrm{a}$ & $2,65 \mathrm{ab}$ & $2,01 \mathrm{a}$ & $1,66 \mathrm{a}$ & $0,82 \mathrm{a}$ \\
CV (\%) & $\mathbf{7 , 3 7}$ & $\mathbf{5 , 9 7}$ & $\mathbf{1 1 , 0 7}$ & $\mathbf{1 5 , 7 1}$ & $\mathbf{6 , 0 1}$ & $\mathbf{1 2 , 9 8}$ & $\mathbf{1 4 , 4 4}$ & $\mathbf{8 , 5 2}$ \\
\hline
\end{tabular}

Dias após a poda .As médias seguidas da mesma letra não diferem estatisticamente entre si,pelo teste de Tukey, a 5\% de probabilidade. Médias de 3 frutos.

TABELA 7 - Relação sólidos solúveis / acidez titulável (SS/AT) de frutos de videira 'Isabel' tratados com elicitores de resistência, no período de setembro de 2009 a janeiro de 2010 (safra 1) e de fevereiro a junho de 2010 (safra 2). Natuba-PB

\begin{tabular}{|c|c|c|c|c|c|c|c|c|}
\hline & \multicolumn{4}{|c|}{ Safra 1} & \multicolumn{4}{|c|}{ Safra 2} \\
\hline & $45^{1}$ & 60 & 90 & 120 & 45 & 60 & 90 & 120 \\
\hline Testemunha & $1,42 \mathrm{a}^{2}$ & $2,14 \mathrm{~b}$ & $3,11 \mathrm{a}$ & $12,00 \mathrm{a}$ & $2,04 \mathrm{a}$ & $2,91 \mathrm{a}$ & $4,77 \mathrm{a}$ & $15,17 \mathrm{a}$ \\
\hline Fungicida & $1,65 \mathrm{a}$ & $2,27 \mathrm{ab}$ & $3,01 \mathrm{a}$ & $15,54 \mathrm{a}$ & $2,28 \mathrm{a}$ & $3,24 \mathrm{a}$ & $5,59 \mathrm{a}$ & $31,26 \mathrm{a}$ \\
\hline Fosfito & $1,59 \mathrm{a}$ & $2,10 \mathrm{~b}$ & $3,11 \mathrm{a}$ & $17,76 \mathrm{a}$ & $1,86 \mathrm{a}$ & $2,95 \mathrm{a}$ & $4,85 \mathrm{a}$ & $28,21 \mathrm{a}$ \\
\hline Agro-Mós® & $1,65 \mathrm{a}$ & $2,40 \mathrm{ab}$ & $3,11 \mathrm{a}$ & $18,89 \mathrm{a}$ & $2,08 \mathrm{a}$ & $3,33 \mathrm{a}$ & $5,63 \mathrm{a}$ & $23,22 \mathrm{a}$ \\
\hline Fung+Fosf. & $1,47 \mathrm{a}$ & $2,00 \mathrm{~b}$ & $2,58 \mathrm{a}$ & $13,68 \mathrm{a}$ & $1,90 \mathrm{a}$ & $2,68 \mathrm{a}$ & $5,00 \mathrm{a}$ & $24,37 \mathrm{a}$ \\
\hline Fung.+Agro-Mós ${ }^{\circledR}$ & $1,45 \mathrm{a}$ & $2,59 \mathrm{a}$ & $3,24 \mathrm{a}$ & $18,64 \mathrm{a}$ & $1,99 \mathrm{a}$ & $3,38 \mathrm{a}$ & $5,96 \mathrm{a}$ & $35,82 \mathrm{a}$ \\
\hline Fosf.+Agro-Mós ${ }^{\circledR}$ & $1,58 \mathrm{a}$ & $2,30 \mathrm{ab}$ & $2,75 \mathrm{a}$ & $18,70 \mathrm{a}$ & $1,93 \mathrm{a}$ & $3,02 \mathrm{a}$ & $4,46 \mathrm{a}$ & $25,73 \mathrm{a}$ \\
\hline Fung.+Fosf.+ Agro-Mós ${ }^{\circledR}$ & $1,48 \mathrm{a}$ & $2,31 \mathrm{ab}$ & $2,63 \mathrm{a}$ & $15,75 \mathrm{a}$ & $1,85 \mathrm{a}$ & $2,89 \mathrm{a}$ & $5,96 \mathrm{a}$ & $21,06 \mathrm{a}$ \\
\hline CV (\%) & 8,39 & 7,63 & 12,49 & 26,80 & 10,96 & 17,23 & 20,84 & 24,56 \\
\hline
\end{tabular}

Dias após a poda. As médias seguidas da mesma letra não diferem estatisticamente entre si, pelo teste de Tukey,a 5\% de probabilidade. Médias de 3 frutos.

TABELA 8 - pH de frutos de videira 'Isabel' tratados com elicitores de resistência, no período de setembro de 2009 a janeiro de 2010 (safra 1) e de fevereiro a junho de 2010 (safra 2). Natuba-PB

\begin{tabular}{lcccccccc}
\hline & \multicolumn{4}{c}{ Safra 1 } & \multicolumn{6}{c}{ Safra $\mathbf{2}$} \\
\hline & $\mathbf{4 5}$ & $\mathbf{6 0}$ & $\mathbf{9 0}$ & $\mathbf{1 2 0}$ & $\mathbf{4 5}$ & $\mathbf{6 0}$ & $\mathbf{9 0}$ & $\mathbf{1 2 0}$ \\
Testemunha & $2,68 \mathrm{a}$ & $2,58 \mathrm{a}$ & $2,51 \mathrm{ab}$ & $3,04 \mathrm{a}$ & $2,12 \mathrm{a}$ & $2,28 \mathrm{a}$ & $2,45 \mathrm{a}$ & $2,97 \mathrm{a}$ \\
Fungicida & $2,68 \mathrm{a}$ & $2,65 \mathrm{a}$ & $2,66 \mathrm{a}$ & $3,11 \mathrm{a}$ & $2,02 \mathrm{a}$ & $2,45 \mathrm{a}$ & $2,52 \mathrm{a}$ & $2,90 \mathrm{a}$ \\
Fosfito & $2,75 \mathrm{a}$ & $2,58 \mathrm{a}$ & $2,46 \mathrm{~b}$ & $3,10 \mathrm{a}$ & $2,07 \mathrm{a}$ & $2,35 \mathrm{a}$ & $2,45 \mathrm{a}$ & $3,05 \mathrm{a}$ \\
Agro-Mós ${ }^{\circledR}$ & $2,69 \mathrm{a}$ & $2,64 \mathrm{a}$ & $2,55 \mathrm{ab}$ & $3,04 \mathrm{a}$ & $2,18 \mathrm{a}$ & $2,45 \mathrm{a}$ & $2,45 \mathrm{a}$ & $2,98 \mathrm{a}$ \\
Fung+Fosf. & $2,69 \mathrm{a}$ & $2,56 \mathrm{a}$ & $2,50 \mathrm{ab}$ & $3,03 \mathrm{a}$ & $2,02 \mathrm{a}$ & $2,35 \mathrm{a}$ & $2,59 \mathrm{a}$ & $3,00 \mathrm{a}$ \\
Fung.+Agro-Mós ${ }^{\circledR}$ & $2,69 \mathrm{a}$ & $2,59 \mathrm{a}$ & $2,53 \mathrm{ab}$ & $3,15 \mathrm{a}$ & $2,04 \mathrm{a}$ & $2,39 \mathrm{a}$ & $2,43 \mathrm{ab}$ & $3,05 \mathrm{a}$ \\
Fosf.+Agro-Mós ${ }^{\circledR}$ & $2,51 \mathrm{~b}$ & $2,66 \mathrm{a}$ & $2,53 \mathrm{ab}$ & $3,06 \mathrm{a}$ & $2,05 \mathrm{~b}$ & $2,45 \mathrm{a}$ & $2,39 \mathrm{ab}$ & $2,90 \mathrm{a}$ \\
Fung.+Fosf.+Hgro-Mós ${ }^{\circledR}$ & $2,67 \mathrm{a}$ & $2,65 \mathrm{a}$ & $2,53 \mathrm{ab}$ & $2,96 \mathrm{a}$ & $2,07 \mathrm{a}$ & $2,45 \mathrm{a}$ & $2,39 \mathrm{ab}$ & $2,78 \mathrm{a}$ \\
\hline CV (\%) & $\mathbf{2 , 2 5}$ & $\mathbf{3 , 0 6}$ & $\mathbf{2 , 7 2}$ & $\mathbf{2 , 7 2}$ & $\mathbf{3 , 5 6}$ & $\mathbf{4 , 1 5}$ & $\mathbf{3 , 2 5}$ & $\mathbf{4 , 3}$ \\
\hline
\end{tabular}

* As médias seguidas da mesma letra não diferem estatisticamente entre si,pelo teste de Tukey, a 5\% de probabilidade. Médias de 3 frutos. 

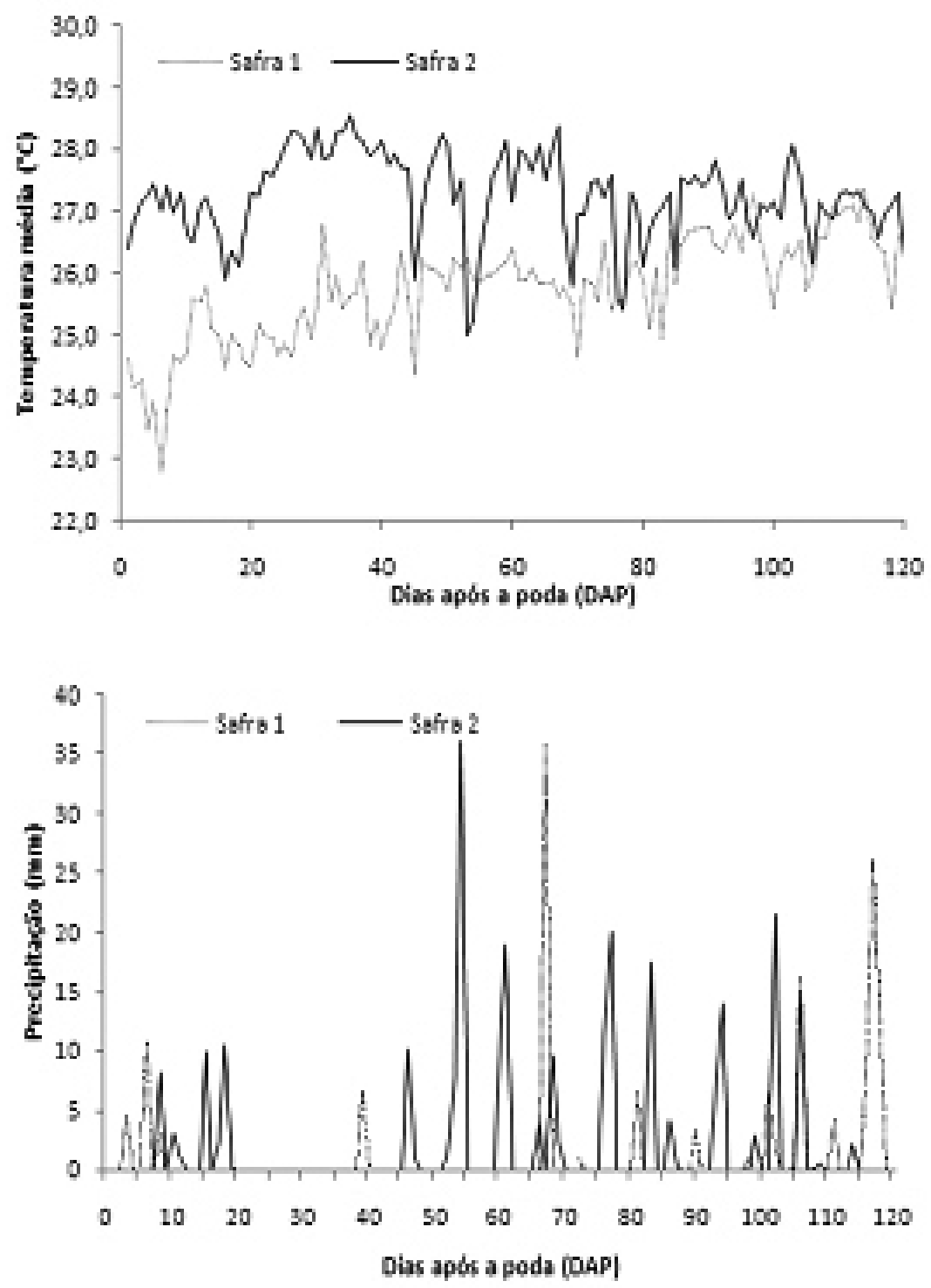

FIGURA 1- Dados diários de temperatura e precipitação do município de Natuba-PB, nos períodos de setembro de 2009 a janeiro de 2010 (safra 1) e de fevereiro a junho de 2010 (safra 2).

\section{CONCLUSÕES}

1-A massa e o comprimento dos cachos de uva 'Isabel' são influenciados positivamente pela aplicação de fosfito de potássio na safra 1.

2-Os elicitores de resistência (Fosfito de Potássio e AgroMós ${ }^{\circledR}$ ), aplicados isoladamente ou em conjunto, não interferem no rendimento de polpa nem no índice de colheita de uvas 'Isabel'.

\section{REFERÊNCIAS}

ASSISTAT. Versão 7.5 beta, Campina Grande - PB, 2010

BLOUIN, J.; GUIMBERTEAU, G. Maduración y madurez de la uva. Madrid: Mundi-Prensa, 2004. 
DAVID, V.; YINONG, Y.; CASIANA, V.C.; MONICA, H.O. Abscisic Acid-Induced Resistance against the Brown Spot Pathogen Cochliobolus miyabeanus in Rice Involves MAP Kinase-Mediated Repression of Ethylene Signaling. Plant Physiology, Minneapolis, v. 152, p. 2036-2052, 2010.

DIAS, G.B.; RANGEL, T.B.A. Indução de resistência em plantas: o papel do óxido nítrico. Revista Capixaba de Ciência e Tecnologia, Natal, n.3, p. $1-8,2007$.

DIAS-ARIEIRA，C.R.; FERREIRA，L.R.; ARIEIRA, J.O. Atividade do óleo de Eucalyptus citriodora e Azadirachta indica no controle de Colletotrichum acutatum em morangueiro. Summa Phytopathologica, Botucatu, v.36, p. 228-232, 2010.

EMBRAPA - Empresa Brasileira de Pesquisa Agropecuária. Embrapa Uva e Vinho. Sistemas de produção. Santa Cruz do Sul: Gazeta, 2006. n.9, p.17-18.

FAO. FAOSTAT - statistical data bases. Roma: World Agricultural Information Centre, 2009. Disponível em: $<$ http://apps.fao.org $>$. Acesso em: 06 out. 2011.

GOMES, E. C. S. Indução De Resistência Em Videira (Vitis LABRUSCA L.) NO MUNICÍPIO DE Natuba, Paraíba: produtividade e perfil de maturação. 2009. Dissertação (Mestrado em Agronomia) Universidade Federal da Paraíba, João Pessoa, 2009.

HALL, D.; KIM, K. H. DE LUCA., V. Molecular cloning and biochemical characterization of three Concord grape (Vitis labrusca) flavonol 7-O-glucosyltransferases. Planta, Berlin, v. 234, n.1, p. 1201-1214, 2011.

HERNANDES, J. L.; JÚNIOR, M. J. P.; SANTOS, A. O.; TECCHIO, M. A. Fenologia e produção de cultivares americanas e híbridas de uvas para vinho, em Jundiaí-SP. Revista Brasileira de Fruticultura, Jaboticabal, v. 32, n.1, p. 135-142, 2010.
IBGE, SIDRA. Disponível em: <www.sidra.ibge. gov.br/bda>. Acesso em 06 jun. 2011.

MALACRIDA, C. R.; MOTTA, S. Antocianina em suco de uva: composição e estabilidade. Boletim do CEPPA, Curitiba, v. 24, n. 1, p. 59-82, 2006.

MASCARENHAS, R. J.; SILVA, S. M.; LOPES, J. D.; LIMA, M. A. L. Avaliação sensorial de uvas de mesa produzidas no Vale do São Francisco e comercializadas em João Pessoa-PB. Revista Brasileira de Fruticultura, Jaboticabal, v. 32, n. 4, p. 993-1000, 2010.

OLIVEIRA, A. F. Produtividade da soja e severidade de ferrugem asiática (Phakopsora pachyrhizi) influenciadas pela aplicação foliar com fontes de potássio e doses de tebuconazole. 2007. 51 f. Dissertação (Mestrado em Agronomia) - Universidade Federal de Uberlândia, Uberlândia, 2007.

RIZZON, L. A.; SGANZERLA, A. Àcidos tartárico e málico no mosto de uva Bento Gonçalves-RS. Ciência Rural, Santa Maria, v. 37, n. 3, p. 911-914, 2007.

ROSA, R. C. T.; COELHO, R. S. B.; TAVARES, S. C. C. H.; CAVALCANTI, V. A. L. B. Efeito de indutores no controle de míldio em Vitis labrusca. Summa Phytopathologica, Botucatu, v. 33, n. 1, p. 68-73, 2006.

SATO, A. J.; SILVA, B. J.; BERTOLUCCI, R.; CARIELO, M.; GUIRAUD, M. C.; FONSECA, I. C. B.; ROBERTO, S. F. Evolução da maturação e características físico-químicas da cultivar Isabel sobre diferentes porta-enxertos na Região Norte do Paraná. Ciências Agrárias, Londrina-PA, v. 30, n. 1, 2009, p. 11-20.

TAIZ, L.; ZEIGER, E. Fisiologia vegetal. Tradução de Santarem et al. 3.ed. Porto Alegre: Artmed, 2006. 719 p. 